\title{
Reduced Levels of miR-342-5p in Plasma Are Associated With Worse Cognitive Evolution in Patients With Mild Alzheimer's Disease
}

\begin{abstract}
Farida Dakterzada ${ }^{1 \dagger}$, Iván David Benítez ${ }^{2,3 t}$, Adriano Targa ${ }^{2,3}$, Albert Lladó4, Gerard Torres'2, Leila Romero', David de Gonzalo-Calvo², Anna Moncusi-Moix ${ }^{2,3}$, Adria Tort-Merino ${ }^{4}$, Raquel Huerto ${ }^{1}$, Manuel Sánchez-de-la-Torre ${ }^{2,5}$, Ferran Barbé2,3 and Gerard Piñol-Ripoll'*

${ }^{1}$ Unitat Trastorns Cognitius, Clinical Neuroscience Research, Santa Maria University Hospital, IRBLleida, Lleida, Spain, ${ }^{2}$ Translational Research in Respiratory Medicine, Hospital Universitari Arnau de Vilanova-Santa Maria, IRBLleida, Lleida, Spain, ${ }^{3}$ Centro de Investigación Biomédica en Red de Enfermedades Respiratorias (CIBERES), Madrid, Spain, ${ }^{4}$ Alzheimer's Disease and Other Cognitive Disorders Unit, Neurology Department, Hospital Clínic, Institut D'Investigacion Biomèdiques August Pi i Sunyer, Barcelona, Spain, ${ }^{5}$ Group of Precision Medicine in Chronic Diseases, Hospital Universitari Arnau de Vilanova-Santa Maria, IRBLleida, Lleida, Spain
\end{abstract}

Background: Progressive cognitive decline is the most relevant clinical symptom of Alzheimer's disease (AD). However, the rate of cognitive decline is highly variable
OPEN ACCESS

Edited by:

Ana l. Duarte,

University of Coimbra, Portugal

Reviewed by:

Tong Li,

Johns Hopkins University, United States

Zhonghua Dai,

University of Southern California,

United States

${ }^{*}$ Correspondence:

Gerard Piñol-Ripoll gerard_437302@hotmail.com

tThese authors have contributed equally to this work

Received: 06 May 2021 Accepted: 26 July 2021 Published: 23 August 2021

Citation:

Dakterzada F, David Benítez I, de Gonzalo-Calvo $D$,

Moncusí-Moix A, Tort-Merino A, Barbé F and Piñol-Ripoll G (2021)

Reduced Levels of miR-342-5p

in Plasma Are Associated With Worse Cognitive Evolution in Patients With Mild Alzheimer's Disease. Front. Aging Neurosci. 13:705989. doi: 10.3389/fnagi.2021.705989 Targa A, Lladó A, Torres G, Romero L, Huerto R, Sánchez-de-la-Torre M, between patients. Synaptic deficits are the neuropathological event most correlated with cognitive impairment in $\mathrm{AD}$. Considering the important role of microRNAs (miRNAs) in regulating synaptic plasticity, our objective was to identify the plasma miRNAs associated with the rate of cognitive decline in patients with mild $A D$.

Methods: We analyzed 754 plasma miRNAs from 19 women diagnosed with mild AD using TaqMan low-density array cards. The patients were grouped based on the rate of decline in the MMSE score after 2 years $[<4$ points $(N=11)$ and $\geq 4$ points $(N=8)]$. The differentially expressed miRNAs between the two groups were validated in an independent cohort of men and women $(N=53)$ with mild AD using RT-qPCR.

Results: In the discovery cohort, 17 miRNAs were differentially expressed according to the fold change between patients with faster declines in cognition and those with slower declines. miR-342-5p demonstrated differential expression between the groups and a good correlation with the rate of cognitive decline in the validation cohort $(r=-0.28$; $p=0.026$ ). This miRNA had a lower expression level in patients who suffered from more severe decline than in those who were cognitively more stable after 2 years $(p=0.049)$.

Conclusion: Lower levels of miR-342-5p in plasma were associated with faster cognitive decline in patients with mild AD after 2 years of follow-up.

Keywords: Alzheimer's disease, cognitive decline, miRNA, miR-342-5p, biomarker

\section{INTRODUCTION}

Alzheimer's disease (AD) is an irreversible, progressive brain disorder that gradually destroys memory and other thinking skills and, eventually, leads to complete dependency in daily life activities (Long and Holtzman, 2019). The rate of cognitive decline is highly variable among patients, with some having a faster course than others. Studies have demonstrated a strong 
association between the rate of cognitive decline and mortality in $\mathrm{AD}$ patients (Hui et al., 2003). In addition, understanding the physiopathological processes underlying this variability is highly important because of the great potential benefits for the development of effective therapeutic approaches.

Extracellular amyloid plaques (accumulation of amyloid$\beta$ (A $\beta)$ protein) and intracellular neurofibrillary tangles (aggregations of hyperphosphorylated tau protein, P-tau) are two main pathological hallmarks of AD. Although both of these pathological characteristics are considered specific to $\mathrm{AD}$, none of them have demonstrated a good correlation with the clinical symptoms (Jack et al., 2018). For example, the accumulation of $A \beta$ plaques, known as the first pathological event of $\mathrm{AD}$ according to the "amyloid cascade hypothesis," peaks at the asymptomatic stage of the disease (Selkoe and Hardy, 2016). It is widely accepted that neuronal injury, particularly synaptic loss, is the $\mathrm{AD}$ neuropathological alteration that most correlates with cognitive dysfunction (Jack et al., 2018; Colom-Cadena et al., 2020). The measurement of some proteins released in CSF, such as tau and neurofilament light chain, can be used to assess neurodegeneration and has shown a good correlation with cognitive decline (Jack et al., 2018; Preische et al., 2019). However, they are not specific to neuronal damage due to $\mathrm{AD}$. In addition, the method for obtaining CSF is invasive, which limits its use for the concurrent monitoring of therapeutic trials and drug efficacy and for longitudinal studies where multiple lumbar punctures are needed. Therefore, searching for new biomarkers in the circulatory system that can predict the rate of cognitive decline and reveal neuropathological alterations specific to $\mathrm{AD}$ is of great importance.

MicroRNAs (miRNAs) are small (typically $22 \mathrm{nt}$ in size) noncoding RNA molecules that regulate the activity of messenger RNA targets by binding to their specific binding sites located in the coding domain sequence or $3^{\prime}$-untranslated regions. This union suppresses the translation of the mRNA or induces its degradation (Brümmer and Hausser, 2014). miRNAs are present in tissues and bodily fluids and play important roles in a wide range of physiological and pathological processes, including $\mathrm{AD}$ (Cogswell et al., 2008; Hébert et al., 2008; Boissonneault et al., 2009). Circulatory miRNAs have shown high stability (Mitchell et al., 2008; Turchinovich et al., 2011), making them ideal biomarker targets. Increasing evidence indicates that miRNAs play a pivotal role in the regulation of synapses and synaptic plasticity (Hu and Li, 2017). Therefore, miRNAs are importantly involved in cognitive functions such as learning and memory (Wang et al., 2012). On the other hand, some studies have revealed that deregulation of several miRNAs contributes to synaptic and memory deficits in $\mathrm{AD}$ mouse models (Liu et al., 2017; Wang et al., 2018; Song et al., 2019).

In the present study, we aimed to detect and validate baseline circulating miRNAs that can be associated with the rate of cognitive decline in patients with $\mathrm{AD}$ after 2 years of followup. To this end, we selected a discovery cohort of women with mild $\mathrm{AD}$ and assessed their cognitive loss during a 2-year follow-up by the Mini-Mental State Examination (MMSE). The miRNAs present in the plasma of these patients were subjected to high-throughput miRNA expression profiling. Subsequently, the candidate miRNAs were validated in an independent cohort of men and women with mild AD via individual RT-qPCR methods.

\section{MATERIALS AND METHODS}

\section{Study Population}

The subjects were prospectively recruited from a sample of outpatients who visited the Cognitive Disorders Unit at Hospital Universitari Santa Maria in Lleida and Hospital Clínic de Barcelona. The discovery cohort consisted of 19 women diagnosed with mild AD (MMSE score $\geq 20$ ) and with abnormal A $\beta 42$ levels $(\leq 600 \mathrm{pg} / \mathrm{mL})$ from Hospital Universitari Santa Maria in Lleida. The validation study consisted of 53 subjects with mild $\mathrm{AD}$ and abnormal $\mathrm{A} \beta 42$ levels from Hospital Universitari Santa Maria in Lleida $(N=41)$ and Hospital Clínic de Barcelona $(N=12)$. AD was diagnosed according to the clinical criteria of the National Institute on Ageing and Alzheimer's disease Association (McKhann et al., 2011). Patients with cognitive impairment caused by other conditions, such as stroke, brain tumor, other neurodegenerative diseases, etc. were excluded from the study. We also excluded male patients from the discovery cohort to eliminate the sex effect in this small population.

Demographic data and general medical aspects such as hypertension, diabetes mellitus, hypercholesterolemia, stroke, depression, and APOE4 status were also evaluated in all subjects.

The cognitive evolution of the patients was measured by MMSE at baseline and after one and 2 years. The MMSE is a screening questionnaire for the detection of cognitive impairment (Folstein et al., 1975). It has 30 questions, and each question to be answered is scored with points, with a maximum possible score of 30 points. This questionnaire can be used to estimate the severity of cognitive impairment and to follow the course of cognitive changes in an individual over time. Although the rate of progression is variable among patients with mild $\mathrm{AD}$, there is not any consensus regarding the definition of patients with fast decline versus slow decline. In a study by Stanley and collaborators, a 1.4 point/year $(\approx 3$ points/2 years) decline in MMSE was reported in patients aged $>74$ years (Stanley et al., 2019). Therefore, based on the rate of cognitive decline, we divided each cohort into two groups: patients who had lost less than 4 points (named slow decline in cognition, SDC) and those who had lost four or more points (named fast decline in cognition, FDC) after a 2 -year followup.

\section{Sample Collection, RNA Extraction and Reverse Transcription}

Blood samples were collected at baseline by venipuncture into EDTA-containing tubes between 8:00 and 10:00 A.M. in fasting condition. The samples were centrifuged at $2500 \mathrm{~g}$ for $10 \mathrm{~min}$, and plasma was separated, aliquoted and stored at $-80^{\circ} \mathrm{C}$ until use. Total RNA was extracted from plasma samples by using the mirVana PARIS RNA and Native Protein Purification Kit (Cat. No. AM1556, Thermo Fisher 
Scientific) according to the manufacturer's instructions. Isolation of total RNA instead of small RNAs was recommended by the manufacturer for miRNA expression profiling using miRNA arrays. Briefly, $300 \mu \mathrm{l}$ plasma was added to an equal volume of $2 \times$ denaturing solution and then spiked with $10 \mu \mathrm{l}$ of $100 \mathrm{pM}$ synthetic cel-miR-39-3p (478293_mir, Thermo Fisher Scientific). The cel-miR-39-3p was added to the samples as an external control in order to monitor the RNA extraction efficiency. The phenol extraction was applied, and finally, total RNA was eluted in $40 \mu \mathrm{l}$ of $95^{\circ} \mathrm{C}$ nuclease-free water following the recommended protocol. Two microlitres of RNA was reverse-transcribed to cDNA template and amplified using the TaqMan Advanced miRNA cDNA Synthesis Kit (Cat No. A25576, Applied Biosystems) and according to the corresponding user guide (publication number MAN0016122, revision C.0). The amplified cDNA product was stored at $-20^{\circ} \mathrm{C}$ until use.

\section{Profiling of miRNAs Using TaqMan Low-Density Array (TLDA) Cards}

The expression profiling of miRNAs in 19 plasma samples was carried out by loading a 1:10 dilution of amplified cDNA and TaqMan Fast Advanced Master Mix into two microarray cards (TaqMan Advanced miRNA Human A and B cards, Applied Biosystems), each containing 384 assays. The cards were run on a QuantStudio 7 Flex RTPCR system (Life Technologies) and amplified based on the corresponding user guide (publication number MAN0016122, revision C.0). The threshold values were determined by QuantStudio software v-1.3. The data were processed with the Relative Quantification tool (powered by Thermo Fisher cloud), and their quality was evaluated based on the following criteria: (1) RT-PCR products were considered below the detection threshold and deleted if $\mathrm{Ct} \geq 35$ or if the $\mathrm{Ct}$ value were reported as "Undetected" and (2) RT-PCR products with an acceptable $\mathrm{Ct}$ range but an irregular amplification curve was censored. After evaluation of the quality of the raw data (Supplementary Figures 1, 2), they were normalized based on the mean-centering method, which is the gold standard when a high number of miRNAs are evaluated (Wylie et al., 2011; Faraldi et al., 2019).

\section{Validation of Differentially Expressed miRNAs by RT-qPCR}

The differentially expressed miRNAs from the microarray experiment were validated in a new and independent cohort that consisted of 53 AD patients. RT-qPCR was carried out by using individual TaqMan Advanced miRNA Assays and TaqMan Fast Advanced Master Mix that were loaded on 384-well plates (Applied Biosystems). The samples were run in duplicate for each assay. We normalized these data using four endogenous controls (EC) (miR103a-2-5p, miR-22-5p, miR-1301-3p, and miR-425-3p) and cel-miR-39 as an exogenous control. These ECs were shown to be stable in the plasma samples of controls and patients with or without pathophysiological changes in $\mathrm{AD}$ (Dakterzada et al., 2020).

\section{Target Analysis}

We used TargetScan ${ }^{1}$ and miRDB $^{2}$ tools to search for the possible target genes of the differentially expressed miRNAs. The biological targets of microRNAs in TargetScan are predicted by searching for the presence of sites that match the seed region of each miRNA (Bartel, 2009). In miRDB, miRNA targets are predicted from interactive modeling of miRNA binding and overexpression data (Liu and Wang, 2019).

\section{Ethics Approval}

The Clinical Investigation Ethical Committee (CEIC P16/109) of Arnau de Vilanova University Hospital of Lleida approved this study for the discovery cohort. All patients included in the confirmatory cohort signed an internal written regulatory document stating that residual samples used for diagnostic procedures can be used for research studies.

\section{Statistical Analysis}

Quantitative variables are shown as the mean (standard deviation) or median [interquartile range] according to the normality of the data. Absolute and relative frequencies were used to describe qualitative variables. We compared patient characteristics according to the study groups (FDC and SDC) in the discovery and validation cohorts. The $t$-test (or nonparametric Wilcoxon signed-rank test) was used to compare quantitative variables, and the chi-squared test was used for qualitative variables. The differences in miRNA expression between groups were evaluated using linear models for arrays (Ritchie et al., 2015). Given the age differences between study groups in the validation cohort, the linear models were ageadjusted in this cohort. The $p$-value threshold defining statistical differential expression was set at $<0.05$. miRNAs with significant difference between groups and a minimum fold change of 1.25 (or 0.8 for downregulated miRNAs) were considered as differentially expressed. All statistical analyses and data processing procedures were performed using $\mathrm{R}$ software, version 3.5.2 (Vienna, Austria).

\section{RESULTS}

\section{Patient Characteristics}

The discovery and validation cohorts consisted of 19 and 53 patients with mild $\mathrm{AD}$, respectively. In this regard, the pilot study consisted of 11 patients with SDC and 8 patients with FDC, while the validation cohort consisted of 32 SDC and 21 FDC patients. In the validation cohort, patients in the FDC group were older than those in the SDC group. There was no other significant difference regarding demographic data, comorbidities, AD core biomarker level

\footnotetext{
${ }^{1}$ http://www.targetscan.org/vert_72/

${ }^{2}$ www.mirdb.org
} 
TABLE 1 | Characteristics of the study population that participated in the discovery and validation cohorts.

\begin{tabular}{|c|c|c|c|c|c|c|}
\hline & \multicolumn{3}{|c|}{ Discovery cohort } & \multicolumn{3}{|c|}{ Validation cohort } \\
\hline & $\operatorname{SDC}(-4,2]$ & FDC $[-9,-4]$ & $p$-value & & FDC $[-16,-4]$ & $p$-value \\
\hline & $N=11$ & $N=8$ & & $N=32$ & $N=21$ & \\
\hline \multicolumn{7}{|l|}{ Demographic data } \\
\hline Sex, Female & $11(100 \%)$ & $8(100 \%)$ & & $10(31.2 \%)$ & $11(52.4 \%)$ & 0.211 \\
\hline Age & $74.1(5.43)$ & $76.8(6.02)$ & 0.338 & 74.5 [71.5;80.0] & $71.0[66.1 ; 75.0]$ & 0.038 \\
\hline \multicolumn{7}{|l|}{ Comorbidities } \\
\hline Hypertension & $9(81.8 \%)$ & $4(50.0 \%)$ & 0.319 & $19(59.4 \%)$ & $10(47.6 \%)$ & 0.576 \\
\hline Stroke & $2(18.2 \%)$ & $0(0.00 \%)$ & 0.485 & $1(3.12 \%)$ & $1(4.76 \%)$ & 0.999 \\
\hline Diabetes mellitus & $2(18.2 \%)$ & $1(12.5 \%)$ & 1000 & 7 (21.9\%) & $4(19.0 \%)$ & 0.999 \\
\hline Dyslipidemia & $4(36.4 \%)$ & $4(50.0 \%)$ & 0.658 & $12(37.5 \%)$ & $8(38.1 \%)$ & 0.999 \\
\hline Depression & 5 (45.5\%) & $2(25.0 \%)$ & 0.633 & 10 (31\%) & $6(28 \%)$ & 0.861 \\
\hline APOE4 carrier, Yes & $6(54.5 \%)$ & $5(62.5 \%)$ & 0.999 & $13(54.2 \%)$ & $10(55.6 \%)$ & 0.921 \\
\hline \multicolumn{7}{|c|}{ Alzheimer's parameters } \\
\hline Basal MMSE & $21.3(2.53)$ & $24.5(2.62)$ & 0.017 & $23.0(2.92)$ & $23.8(3.11)$ & 0.348 \\
\hline 1-year MMSE & $22.2(1.99)$ & $22.2(1.98)$ & 0.942 & $22.4(3.67)$ & 19.7 (3.84) & 0.016 \\
\hline 2-year MMSE & $19.8(2.71)$ & $18.6(2.72)$ & 0.36 & $22.1(3.44)$ & 16.7 (4.99) & $<0.001$ \\
\hline $\mathrm{A} \beta 42$ & $453(116)$ & $471(86.0)$ & 0.689 & $419(100)$ & $461(106)$ & 0.162 \\
\hline T-tau & $601[501 ; 772]$ & 594 [407;688] & 0.563 & 430 [306;692] & $618[456 ; 861]$ & 0.094 \\
\hline P-tau & $89.6[76.8 ; 118]$ & $93.3[78.8 ; 106]$ & 0.804 & $66.8[51.5 ; 94.6]$ & $87.0[75.7 ; 110]$ & 0.161 \\
\hline
\end{tabular}

SDC, slow decline in cognition; FDC, fast decline in cognition; MMSE, Mini-Mental State Examination; Aß42, Amyloid beta 42; T-tau, Total tau protein; P-tau, Phosphorylated tau protein. P-values were calculated by comparing FDC and SDC patients using t-test (or non-parametric Wilcoxon signed-rank test) for continuous variables and chi-squared test for categorical variables.

or MMSE at baseline between patients included in each cohort (Table 1).

\section{Identification of miRNAs Related to the Rate of Cognitive Impairment (TLDA Experiment)}

We identified 17 miRNAs that were differentially expressed between the two groups (Figure 1A). The expression profile of these 17 miRNAs was able to completely discriminate between the two groups of the study (Figure 1B). Among these 17 miRNAs, miR-25-3p (0.53 fold change), miR-496 ( 0.15 fold change), miR-342-5p (0.33 fold change), miR-193a-3p (0.1 fold change), miR-483-5p (0.5 fold change), and let-7c$5 \mathrm{p}$ (0.04 fold change) were downregulated in the FDC group (Figure 1A, red dots and Supplementary Table 1). Eleven miRNAs, including miR-30e-5p (2.01 fold change), miR-153-3p (1.69 fold change), miR-497-5p (1.76 fold change), miR-196b-3p (4.97 fold change), miR-148a-5p (2.83 fold change), miR-191-3p (1.76 fold change), miR-652-3p (1.59 fold change), miR-431$3 p$ (3.01 fold change), miR-30d-3p (8.22 fold change), miR744-3p (4.34 fold change), and miR-27b-5p (1.99 fold change), were upregulated in the FDC group (Figure 1A, blue dots and Supplementary Table 1). Furthermore, all 17 miRNAs had a good correlation with the rate of cognitive decline (Supplementary Table 1).

From these 17 miRNAs, 16 were selected for validation in an independent cohort. The sequences of all 17 miRNAs are shown in Supplementary Table 1. We eliminated the miRNA miR-193a-3p from the list of validation because it had a similar expression pattern between two groups except for 3 outlier patients in SDC group that had a lower expression than the other members of group.

\section{Validation of Differentially Expressed miRNAs}

Individual RT-qPCR probes were used for validation of the 16miRNA signature in an independent cohort of patients with mild $\mathrm{AD}(N=53)$. After evaluation of the quality of the data (Supplementary Figure 3) and normalization, as explained in the Methods section, miR-342-5p showed significant differences in expression between the SDC and FDC groups (Supplementary Figure 4). Then, we dichotomized the patients into high and low expression of miR-342-5p groups, and our results revealed that patients with low expression of miR-342-5p had a worse cognitive evolution after 2 years of follow-up $(p=0.026)$ (Figure 2).

\section{Predicted Target Genes for miR-342-5p}

We investigated the potential role of miR-342-5p in the pathological processes of memory loss in $\mathrm{AD}$ by searching through its predicted targets. Interestingly, not only proteins directly related to $\mathrm{AD}$, such as beta-site amyloid precursor protein cleaving enzyme 1 , microtubule associated protein $1 \mathrm{~A}$ and tau tubulin kinase 1 , but also the genes for many synaptic proteins were among targets that may be regulated by this miRNA (Table 2). Importantly, the ephrin A2 gene had the best target rank in miRDB and the second best target rank in TargetScan for miR-342-5p. 


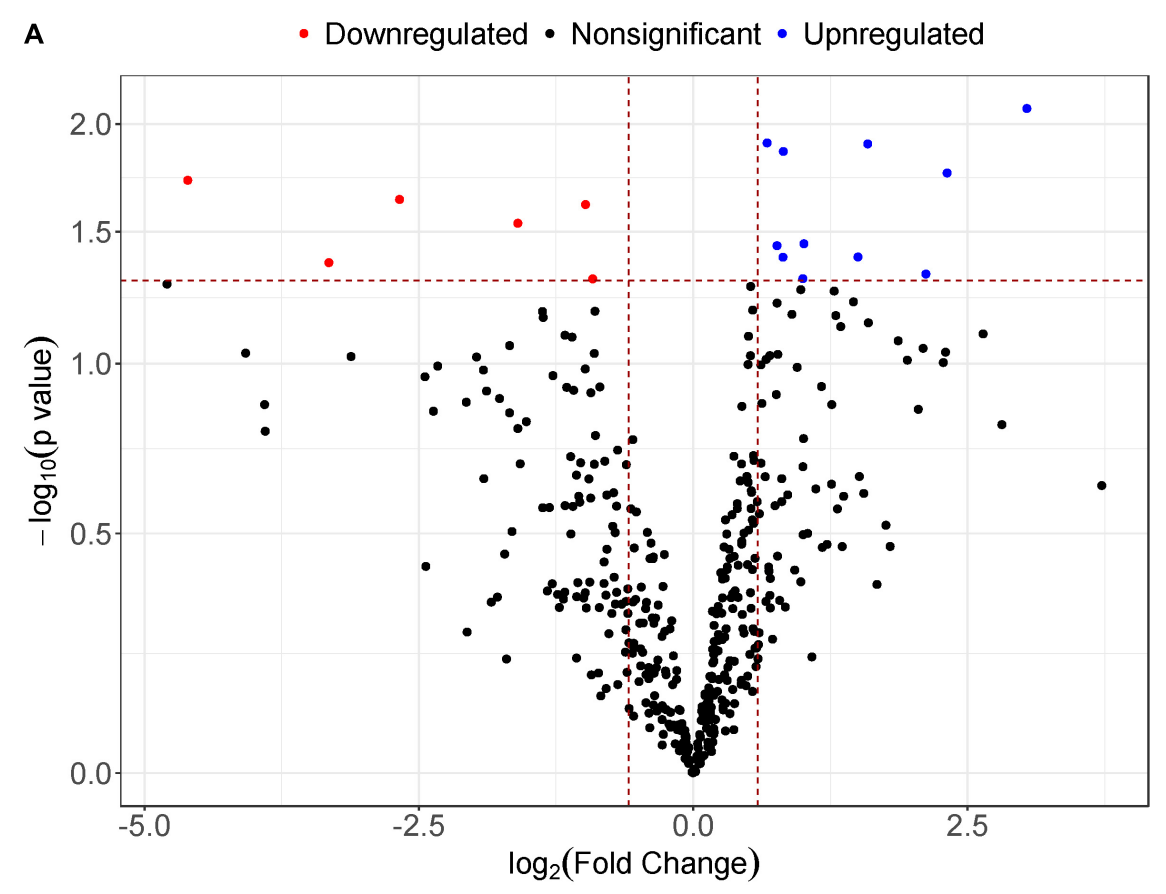

B

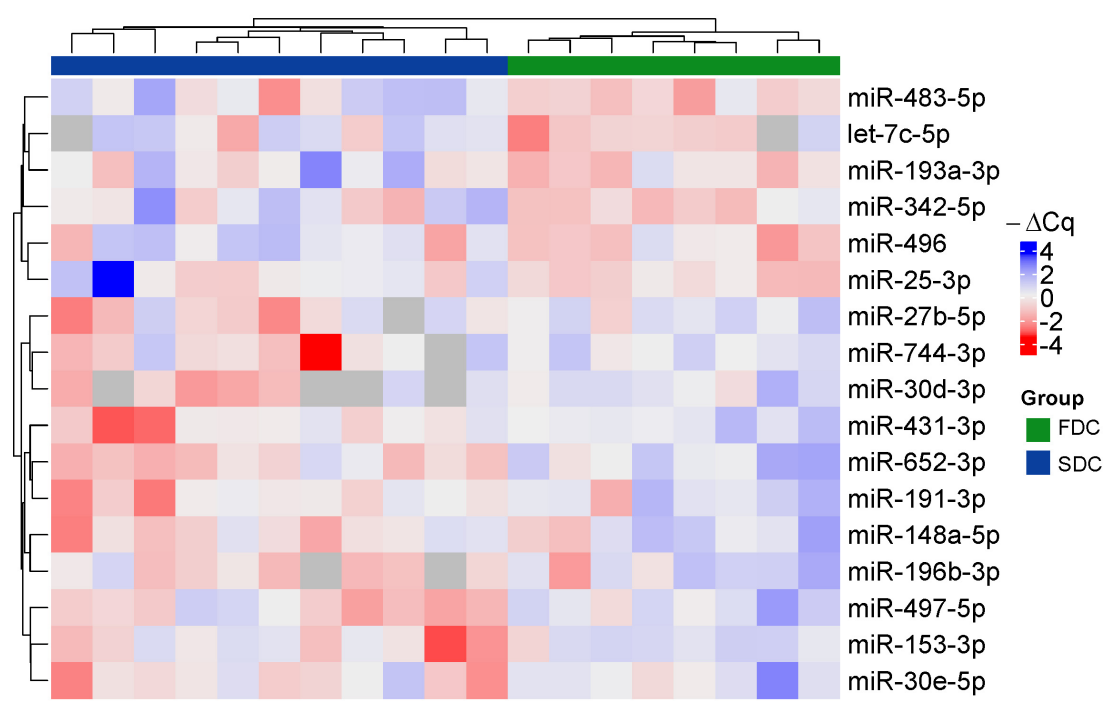

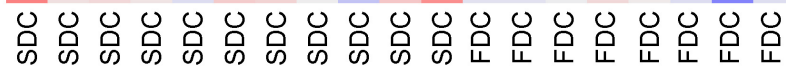

FIGURE 1 | (A) Volcano plot of the distribution of 17 differentially expressed miRNAs at baseline between patients with FDC and SDC in the discovery study, mapping six downregulated miRNAs (red dots) and 11 upregulated miRNAs (Blue dots) in the FDC group; (B) Heatmap of all differentially expressed miRNAs at baseline between AD patients with FDC or SDC in the discovery cohort. Red is low expression and blue is high expression.

\section{DISCUSSION}

This study was designed to detect the association of miRNAs with the rate of cognitive decline measured by the MMSE in patients with mild $\mathrm{AD}$ using a hypothesis-free approach. We identified 17 miRNAs that were differentially expressed between women with $\mathrm{AD}$ who suffered from a faster cognitive decline over 2 years and those with slower cognitive decline. We validated these results in an independent cohort of men and women with AD. Our results revealed that miR-342-5p had a good correlation with the rate of cognitive decline, and the patients with lower expression levels of this miRNA had worse cognitive evolution after 2 years of followup.

Importantly, many differential miRNAs in the discovery study were previously associated with AD. For instance, miR-431$3 p$ has been reported to prevent $A \beta$-mediated synaptic loss (Ross et al., 2018), and let-7c-5p, miR-483-5p, miR-342-5p, and miR-191-3p have been associated with $A D$ in several studies 


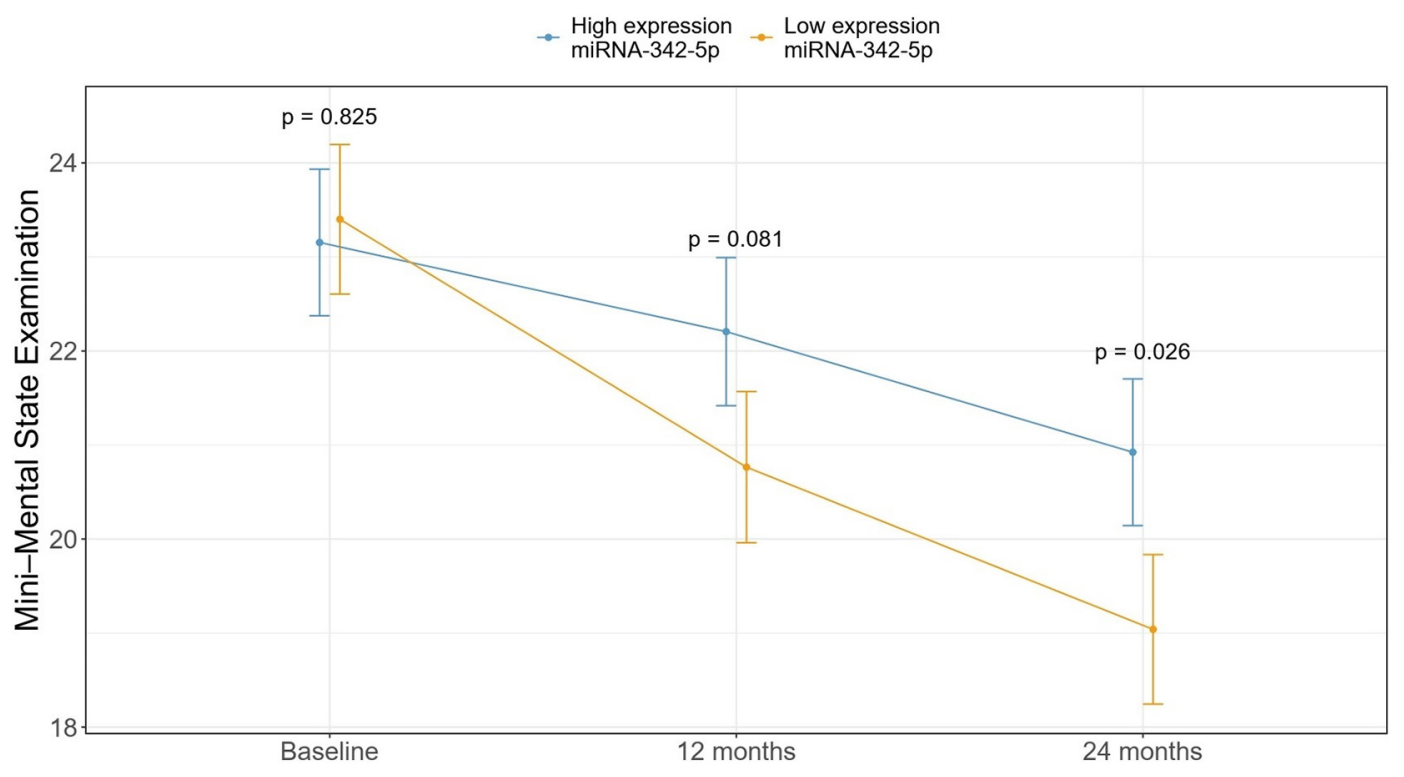

FIGURE 2 | The association between the mean MMSE score in patients with high and low expression of miR-342-5p at baseline and after 1 and 2 years of follow-up. Patients with higher expression of miR-342-5p at baseline showed a slower cognitive deterioration after 2 years of follow-up compared with patients with low expression of this miRNA.

(Takousis et al., 2019). In addition, miR-153-3p was reported to inhibit the expression of amyloid precursor protein (APP) (Liang et al., 2012; Long et al., 2012).

It is widely accepted that memory and cognitive impairment in $\mathrm{AD}$ primarily result from synaptic failure. Gene array experiments have shown alterations in genes involved in neurotransmitter receptors and receptor trafficking, synaptic vesicle trafficking and release, cell adhesion regulating synaptic stability, post-synaptic density scaffolding, and neuromodulatory systems in the early stages of AD (Scheff et al., 2007; Chang et al., 2012; Berchtold et al., 2013). Although the association of miRNAs with $\mathrm{AD}$ has been widely studied, there is scarce information about the miRNAs related to the rate of cognitive impairment and their role in the processes that underlie cognitive decline in these patients. To date, some studies have been conducted to determine the miRNAs important for the regulation of synaptic structure and function and to study their dysregulation and their effect on cognitive impairment in animal models of $\mathrm{AD}$ (Liu et al., 2017; Wang et al., 2018; Song et al., 2019; BarrosViegas et al., 2020). For example, Song et al. (2019) found that upregulation of miR-30b causes synaptic and cognitive deficits in 5XFAD APP transgenic mice. miR-30b targets molecules such as ephrin type-B receptor 2, sirtuin1, and glutamate receptor subunit 2 that are important for maintaining synaptic integrity. These investigators observed that WT mice treated with miR$30 \mathrm{~b}$ showed impaired spatial learning and memory retention measured by the Morris water maze and novel object recognition tests (Song et al., 2019). In a study by Barros-Viegas and collaborators, overexpression of miR-31-5p in a $3 \times \mathrm{Tg}-\mathrm{AD}$ model resulted in a better performance of animals in the T-maze, novel object recognition and Barnes maze, which were used for assessing spatial memory and short-term and long-term memory, respectively (Barros-Viegas et al., 2020).

To our knowledge, this is the first study in which the association of miRNAs has been evaluated with cognitive evolution in patients with mild AD. In a targeted study by Tan et al. (2014) the serum levels of several miRNAs related to $\mathrm{AD}$ were evaluated in patients with $\mathrm{AD}$ and healthy controls. They observed that serum levels of miR$125 \mathrm{~b}$ had a negative correlation with MMSE score in AD patients (Tan et al., 2014). Wiedrick and collaborators assessed the correlation of the CSF levels of 14 miRNAs that were differentially expressed between AD and controls with MMSE. They observed that in these profiles, miR-193a-5p showed a higher correlation with MMSE (Wiedrick et al., 2019). However, in contrast to our study, in none of these studies assessed the association of miRNAs with the cognitive evolution of $\mathrm{AD}$ during a given time of follow-up. In a study by Mengel-From et al. (2018) the association between plasma miRNAs and MMSE and Cognitive Composite Score (CCS) was evaluated in healthy aged twins followed-up for 10 years. They observed that miR-151a-3p, miR-212-3p, and miR-1274b were associated with CCS in both the individual and paired analyses. miR-548c-3p, miR-539-5p, miR-532-3p, miR-369-3p, miR-548a-3p, and miR-27a-5p were associated with the MMSE score (Mengel-From et al., 2018).

In the present study, we validated the differential expression of plasma miR-342-5p between $\mathrm{AD}$ patients with FDC and SDC. This miRNA had a good correlation with the rate of cognitive decline evaluated by the MMSE. Interestingly, we found the genes for many synaptic proteins, including ephrin A2, syntaxin, synaptotagmin, synaptojanin, and neurogranin, among 
TABLE 2 | Possible target genes of miR-342-5p found in miRDB and TargetScan databases that are associated with AD or synaptic plasticity.

\begin{tabular}{|c|c|c|c|}
\hline Possible target & Gene symbol & $\begin{array}{l}\text { Biological role related to synaptic plasticity and } \\
\text { cognition (reference) }\end{array}$ & Database \\
\hline \multicolumn{4}{|l|}{ AD associated targets } \\
\hline Beta-site APP-cleaving enzyme 1 & BACE 1 & $\begin{array}{l}\text { Breakage of amyloid precursor protein and production } \\
\text { of } A \beta \text { protein (Cole and Vassar, 2007) }\end{array}$ & $M \& T$ \\
\hline Tau tubulin kinase 1 & TTBK1 & Phosphorylation of tau protein (Taylor et al., 2019) & $M \& T$ \\
\hline Microtubule associated protein $1 \mathrm{~A}$ & MAP1A & Stabilization of microtubules (Fifre et al., 2006) & $M \& T$ \\
\hline \multicolumn{4}{|l|}{ Synapse associated targets } \\
\hline Ephrin A2 and A5 & EFNA2 and A5 & $\begin{array}{l}\text { Regulation of contact-dependent cell communication } \\
\text { (Chen et al., 2012) }\end{array}$ & $M \& T$ \\
\hline Ephrin A3 and B1-B3 & EFNA3 and B1-B3 & $\begin{array}{l}\text { Regulation of contact-dependent cell communication } \\
\text { (Chen et al., 2012) }\end{array}$ & $\mathrm{T}$ \\
\hline Eph receptor A8, A10, and B4 & EPHA8, A10, and B4 & $\begin{array}{l}\text { Regulation of contact-dependent cell communication } \\
\text { (Chen et al., 2012) }\end{array}$ & $\mathrm{T}$ \\
\hline Eph receptor A1 & EPHA1 & $\begin{array}{l}\text { Regulation of contact-dependent cell communication } \\
\text { (Chen et al., 2012) }\end{array}$ & M \\
\hline Synaptic Ras GTPase activating protein 1 & SYNGPA1 & $\begin{array}{l}\text { Role in dendritic spine synapse maturation (Clement } \\
\text { et al., 2012) }\end{array}$ & $M \& T$ \\
\hline Insulin like growth factor 2 & IGF2 & $\begin{array}{l}\text { Role in synaptogenesis and spine maturation } \\
\text { (Pascual-Lucas et al., 2014) }\end{array}$ & $M \& T$ \\
\hline Synaptophysin & SYP & $\begin{array}{l}\text { Regulation of synaptic vesicle endocytosis (Kwon and } \\
\text { Chapman, 2011) }\end{array}$ & $\mathrm{T}$ \\
\hline Synaptopodin 2 & SYNPO2 & Role in dendritic spine stabilization (Yap et al., 2020) & $\mathrm{T}$ \\
\hline Synaptosomal-associated protein, 91 kDa & SNAP91 & $\begin{array}{l}\text { Role in synaptic vesicle recycling (Vanlandingham et al., } \\
\text { 2014) }\end{array}$ & $M \& T$ \\
\hline Synaptosomal-associated protein, 47 kDa & SNAP47 & Mediating synaptic vesicle fusion (Urbina et al., 2021) & $\mathrm{T}$ \\
\hline $\begin{array}{l}\text { NMDA receptor synaptonuclear signaling } \\
\text { and neuronal migration factor }\end{array}$ & NSMF & $\begin{array}{l}\text { Regulating synaptic stability and neuronal degeneration } \\
\text { (Karpova et al., 2013) }\end{array}$ & $M \& T$ \\
\hline Synaptic vesicle glycoprotein $2 \mathrm{~A}$ and $2 \mathrm{C}$ & SV2A and $2 \mathrm{C}$ & Role in neurotransmission (Stout et al., 2019) & $\mathrm{T}$ \\
\hline Neurogranin & NRGN & Role in post-synaptic signaling (Díez-Guerra, 2010) & $\mathrm{T}$ \\
\hline Syntaxin 1 & STX1 & Mediating synaptic vesicle fusion (Lam et al., 2008) & $M \& T$ \\
\hline Vesicle-associated membrane protein 2 & VAMP2 & Mediating synaptic vesicle fusion (Lam et al., 2008) & $\mathrm{T}$ \\
\hline Vesicle-associated membrane protein 5 & VAMP5 & Mediating synaptic vesicle fusion (Lam et al., 2008) & $M \& T$ \\
\hline Synaptotagmin 2, 5, 6, 7, 9, 11, and 17 & SYT2, 5-7, 9, 11, and 17 & Mediating synaptic vesicle fusion (Wu et al., 2020) & $\mathrm{T}$ \\
\hline Synaptogyrin 3 & SYNGR3 & Regulation of neurotransmitter release (Raja et al., 2019) & $\mathrm{T}$ \\
\hline Synaptojanin 1 & SYNJ1 & Role in synaptic vesicle recycling (Harris et al., 2000) & $\mathrm{T}$ \\
\hline Synapsin 1 & SYN1 & $\begin{array}{l}\text { Regulation of neurotransmitter release (Cesca et al., } \\
\text { 2010) }\end{array}$ & $\mathrm{T}$ \\
\hline $\begin{array}{l}\text { Regulating synaptic membrane exocytosis } \\
3\end{array}$ & RIMS3 & Regulation of synaptic vesicle fusion (Wang et al., 1997) & $\mathrm{T}$ \\
\hline Bassoon presynaptic cytomatrix protein & BSN & $\begin{array}{l}\text { Organizing neurotransmitter release site (Dresbach } \\
\text { et al., 2003) }\end{array}$ & $\mathrm{T}$ \\
\hline $\begin{array}{l}\text { Glutamate ionotropic receptor NMDA type } \\
\text { subunit } 2 A\end{array}$ & GRIN2A & Neurotransmitter receptor (Paoletti and Neyton, 2007) & M \\
\hline Neurexin 2 & NRXN2 & Trans-synaptic connector (Knight et al., 2011) & $\mathrm{T}$ \\
\hline Complexin 1 & CPLX1 & Mediating synaptic vesicle fusion (Maximov et al., 2009) & $\mathrm{T}$ \\
\hline Complexin 2 & CPLX2 & Mediating synaptic vesicle fusion (Maximov et al., 2009) & $M \& T$ \\
\hline
\end{tabular}

AD, Alzheimer's disease; M, miRDB; T, TargetScan.

possible targets of miR-342-5p. Some of these targets, such as neurogranin, have been shown to have a good correlation with the rate of cognitive decline in patients with $\mathrm{AD}$ (Portelius et al., 2015; Headley et al., 2018). Therefore, it is possible that miR-342$5 \mathrm{p}$ plays a part in the cognitive alteration of $\mathrm{AD}$ via the regulation of some synaptic genes. However, this hypothesis should be tested in cellular experiments and animal models of $\mathrm{AD}$.
miR-342-5p was reported to regulate the proliferation and differentiation of neuronal stem cells (Gao et al., 2017). Sun et al. (2014) reported higher levels of miR-342-5p in APP/PS1, PS1DE9, and PS1-M146 V mouse models than in the wildtype mouse brain and suggested that miR-342-5p plays a role in $\mathrm{AD}$ axonopathy by hampering the function of the axon initial segment via downregulation of ankyrin G. Although it 
is challenging to compare our results with the results of other studies in which different tissues and species and study designs were used, our result is not in accordance with the results presented by Sun et al. (2014) because we detected higher levels of miR-342-5p in patients who were cognitively more stable than those who were suffering from a more severe decline. However, our results are in agreement with the study by Lugli et al. (2015), who reported downregulation of miR-342-5p in plasma exosomal samples of patients with $\mathrm{AD}$ compared with non-demented controls.

This study has several strengths. We identified and validated miRNAs related to the rate of cognitive evolution in two independent cohorts. We only included mild AD patients with pathological levels of $A \beta 42$ in both cohorts to assure that assessed cognitive decline is due to AD. Furthermore, we evaluated a high number of miRNAs in the discovery study to identify all possible candidates. Finally, the cognitive alterations of the patients were followed-up for 2 years, while in previous studies (Tan et al., 2014; Wiedrick et al., 2019), the association of miRNAs was assessed with the cognitive status of the patients at baseline.

This study has some limitations. First, the discovery cohort consisted of only female participants; however, we included male subjects in the validation cohort to overcome the bias that may have been caused by this issue. Second, the number of participants in the both discovery and validation cohorts was small, which may have affected the final result of our study. Third, there was a significant difference regarding baseline MMSE scores between FDC and SDC groups in the discovery cohort. Finally, from 16 differential miRNAs selected for validation, we only validated one miRNA. All aforementioned limitations and variabilities related to the normalization method and analytical platforms between two cohorts may have caused this inconsistency. Therefore, this result does not rule out the importance of other differential miRNAs in the cognitive evolution of AD.

To our knowledge, this is the first study seeking to identify miRNAs related to the rate of cognitive decline in patients with mild $\mathrm{AD}$ among a large profile of miRNAs. We detected 17 miRNAs that were able to perfectly separate $\mathrm{AD}$ patients with FDC from those with SDC. From this panel, we validated that miRNA miR-342-5p was associated with the rate of cognitive decline in an independent cohort, suggesting that uncovering the role of miRNAs in cognition may be of interest in seeking new biomarkers and furthering our understanding of the neuropathological processes underlying cognitive decline in AD.

\section{DATA AVAILABILITY STATEMENT}

The raw data supporting the conclusions of this article will be made available by the authors, without undue reservation.

\section{REFERENCES}

Barros-Viegas, A. T., Carmona, V., Ferreiro, E., Guedes, J., Cardoso, A. M., Cunha, P., et al. (2020). miRNA-31 improves cognition and abolishes amyloid- $\beta$

\section{ETHICS STATEMENT}

The studies involving human participants were reviewed and approved by the Clinical Investigation Ethical Committee (CEIC P16/109) of Arnau de Vilanova University Hospital of Lleida. The patients/participants provided their written informed consent to participate in this study.

\section{AUTHOR CONTRIBUTIONS}

FD, ID, AT, and GP-R designed the study. FD and AM-M carried out the experiments. ID, AT, GP-R, AL, AT-M, FB, and FD analyzed the data. FD, ID, AT, AL, GT, LR, DG-C, AT-M, RH, MS-T, FB, and GP-R interpreted the data. FD, ID, and GP-R wrote the manuscript draft. All authors revised the manuscript and approved it for submission.

\section{FUNDING}

Generalitat of Catalonia, Department of Health (PERIS 2019 SLT008/18/00050) and "Fundació La Marató TV3" (464/C/2014) to GP-R. IRBLleida is a CERCA Programme/Generalitat of Catalonia. FD was supported by Agency for Management of University and Research Grants and European Social Fund (FIB100153). AL received funding from Generalitat of Catalonia, Department of Health (PERIS 2016-2020 SLT008/18/00061) and Spanish Ministry of Science and Innovation - Instituto de Salud Carlos III and Fondo Europeo de Desarrollo Regional (FEDER), European Union ("A way to build Europe") (PI19/00449).

\section{ACKNOWLEDGMENTS}

We would like to express our sincere gratitude to all the patients and all the members of the dementia unit at the Hospital Universitari Santa Maria. We were supported by the IRBLleida Biobank (B.0000682) and PLATAFORMA BIOBANCOS PT17/0015/0027/.

\section{SUPPLEMENTARY MATERIAL}

The Supplementary Material for this article can be found online at: https://www.frontiersin.org/articles/10.3389/fnagi. 2021.705989/full\#supplementary-material

pathology by targeting app and bacel in an animal model of Alzheimer's disease. Mol. Ther. Nucleic Acids 19, 1219-1236. doi: 10.1016/j.omtn.2020.01.010

Bartel, D. P. (2009). MicroRNAs: target recognition and regulatory functions. Cell 136, 215-233. doi: 10.1016/j.cell.2009.01.002 
Berchtold, N., Coleman, P., Cribbs, D., Rogers, J., Gillen, D., and Cotman, C. (2013). Synaptic genes are extensively downregulated across multiple brain regions in normal human aging and Alzheimer's disease. Neurobiol. Aging 34, 1653-1661. doi: 10.1016/j.neurobiolaging.2012.11.024

Boissonneault, V., Plante, I., Rivest, S., and Provost, P. (2009). MicroRNA-298 and microRNA-328 regulate expression of mouse beta-amyloid precursor protein-converting enzyme 1. J. Biol. Chem. 284, 1971-1981. doi: 10.1074/jbc. M807530200

Brümmer, A., and Hausser, J. (2014). MicroRNA binding sites in the coding region of mRNAs: extending the repertoire of post-transcriptional gene regulation. BioEssays 36, 617-626. doi: 10.1002/bies.201300104

Cesca, F., Baldelli, P., Valtorta, F., and Benfenati, F. (2010). The synapsins: key actors of synapse function and plasticity. Prog. Neurobiol. 91, 313-348. doi: 10.1016/j.pneurobio.2010.04.006

Chang, R., Nouwens, A., Dodd, P., and Etheridge, N. (2012). The synaptic proteome in Alzheimer's disease. Alzheimers Dement. 9, 499-511. doi: 10.1016/ j.jalz.2012.04.009

Chen, Y., Fu, A. K., and Ip, N. Y. (2012). Eph receptors at synapses: implications in neurodegenerative diseases. Cell. Signal. 24, 606-611. doi: 10.1016/j.cellsig. 2011.11.016

Clement, J. P., Aceti, M., Creson, T. K., Ozkan, E. D., Shi, Y., Reish, N. J., et al. (2012). Pathogenic SYNGAP1 mutations impair cognitive development by disrupting maturation of dendritic spine synapses. Cell 151, 709-723. doi: 10.1016/j.cell.2012.08.045

Cogswell, J., Ward, J., Taylor, I., Waters, M., Shi, Y., Cannon, B., et al. (2008). Identification of miRNA changes in Alzheimer's disease brain and CSF yields putative biomarkers and insights into disease pathways. J. Alzheimers Dis. 14, 27-41. doi: 10.3233/jad-2008-14103

Cole, S. L., and Vassar, R. (2007). The Alzheimer's disease $\beta$-secretase enzyme. BACE1. Mol. Neurodegener. 2:22. doi: 10.1186/1750-1326-2-22

Colom-Cadena, M., Spires-Jones, T., Zetterberg, H., Blennow, K., Caggiano, A., DeKosky, S. T., et al. (2020). The clinical promise of biomarkers of synapse damage or loss in Alzheimer's disease. Alzheimers Res. Ther. 12:21. doi: 10.1186/ s13195-020-00588-4

Dakterzada, F., Targa, A., Benítez, I. D., Romero-ElKhayat, L., de Gonzalo-Calvo, D., Torres, G., et al. (2020). Identification and validation of endogenous control miRNAs in plasma samples for normalization of qPCR data for Alzheimer's disease. Alzheimers Res. Ther. 12:163. doi: 10.1186/s13195-020-00735-x

Díez-Guerra, F. J. (2010). Neurogranin, a link between calcium/calmodulin and protein kinase C signaling in synaptic plasticity. IUBMB Life. 62, 597-606. doi: 10.1002/iub.357

Dresbach, T., Hempelmann, A., Spilker, C., tom Dieck, S., Altrock, W. D., Zuschratter, W., et al. (2003). Functional regions of the presynaptic cytomatrix protein bassoon: significance for synaptic targeting and cytomatrix anchoring. Mol. Cell. Neurosci. 23, 279-291. doi: 10.1016/s1044-7431(03)00015-0

Faraldi, M., Gomarasca, M., Sansoni, V., Perego, S., Banfi, G., and Lombardi, G. (2019). Normalization strategies differently affect circulating miRNA profile associated with the training status. Sci. Rep. 9:1584. doi: 10.1038/s41598-01938505-x

Fifre, A., Sponne, I., Koziel, V., Kriem, B., Yen Potin, F. T., Bihain, B. E., et al. (2006). Microtubule-associated protein MAP1A, MAP1B, and MAP2 proteolysis during soluble amyloid beta-peptide-induced neuronal apoptosis. Synergistic involvement of calpain and caspase-3. J. Biol. Chem. 281, 229-240. doi: 10.1074/jbc.M507378200

Folstein, M. F., Folstein, S. E., and McHugh, P. R. (1975). "Mini-mental state”. A practical method for grading the cognitive state of patients for the clinician. J. Psychiatr. Res. 12, 189-198. doi: 10.1016/0022-3956(75)90026-6

Gao, F., Zhang, Y. F., Zhang, Z. P., Fu, L. A., Cao, X. L., Zhang, Y. Z., et al. (2017). miR-342-5p regulates neural stem cell proliferation and differentiation downstream to notch signaling in mice. Stem Cell Rep. 8, 1032-1045. doi: 10.1016/j.stemcr.2017.02.017

Harris, T. W., Hartwieg, E., Horvitz, H. R., and Jorgensen, E. M. (2000). Mutations in synaptojanin disrupt synaptic vesicle recycling. J. Cell. Biol. 150, 589-600. doi: $10.1083 /$ jcb.150.3.589

Headley, A., De Leon-Benedetti, A., Dong, C., Levin, B., Loewenstein, D., Camargo, C., et al. (2018). Neurogranin as a predictor of memory and executive function decline in MCI patients. Neurology. 90, e887-e895. doi: 10.1212/WNL. 0000000000005057
Hébert, S. S., Horré, K., Nicolaï, L., Papadopoulou, A. S., Mandemakers, W., Silahtaroglu, A. N., et al. (2008). Loss of microRNA cluster miR-29a/b-1 in sporadic Alzheimer's disease correlates with increased BACE1/beta-secretase expression. Proc. Natl. Acad. Sci. U.S.A. 105, 6415-6420. doi: 10.1073/pnas. 0710263105

$\mathrm{Hu}, \mathrm{Z}$., and Li, Z. (2017). miRNAs in synapse development and synaptic plasticity. Curr. Opin. Neurobiol. 45, 24-31. doi: 10.1016/j.conb.2017.02.014

Hui, J. S., Wilson, R. S., Bennett, D. A., Bienias, J. L., Gilley, D. W., and Evans, D. A. (2003). Rate of cognitive decline and mortality in Alzheimer's disease. Neurology 61, 1356-1361. doi: 10.1212/01.wnl.0000094327.68399.59

Jack, C. R. Jr., Bennett, D. A., Blennow, K., Carrillo, M. C., Dunn, B., Haeberlein, S. B., et al. (2018). NIA-AA Research Framework: Toward a biological definition of Alzheimer's disease. Alzheimers Dement. 14, 535-562. doi: 10.1016/j.jalz. 2018.02.018

Karpova, A., Mikhaylova, M., Bera, S., Bär, J., Reddy, P. P., Behnisch, T., et al. (2013). Encoding and transducing the synaptic or extrasynaptic origin of NMDA receptor signals to the nucleus. Cel. 152, 1119-1133. doi: 10.1016/j.cell. 2013.02.002

Knight, D., Xie, W., and Boulianne, G. L. (2011). Neurexins and neuroligins: recent insights from invertebrates. Mol. Neurobiol. 44, 426-440. doi: 10.1007/s12035011-8213-1

Kwon, S. E., and Chapman, E. R. (2011). Synaptophysin regulates the kinetics of synaptic vesicle endocytosis in central neurons. Neuron 70, 847-854. doi: 10.1016/j.neuron.2011.04.001

Lam, A. D., Tryoen-Toth, P., Tsai, B., Vitale, N., and Stuenkel, E. L. (2008). SNAREcatalyzed fusion events are regulated by Syntaxin1A-lipid interactions. Mol. Biol. Cell. 19, 485-497. doi: 10.1091/mbc.e07-02-0148

Liang, C., Zhu, H., Xu, Y., Huang, L., Ma, C., Deng, W., et al. (2012). MicroRNA153 negatively regulates the expression of amyloid precursor protein and amyloid precursor-like protein 2. Brain Res. 1455, 103-113. doi: 10.1016/j. brainres.2011.10.051

Liu, D., Tang, H., Li, X. Y., Deng, M. F., Wei, N., Wang, X., et al. (2017). Targeting the HDAC2/HNF-4A/miR-101b/AMPK pathway rescues tauopathy and dendritic abnormalities in Alzheimer's disease. Mol. Ther. 25, 752-764. doi: 10.1016/j.ymthe.2017.01.018

Liu, W., and Wang, X. (2019). Prediction of functional microRNA targets by integrative modeling of microRNA binding and target expression data. Genome Biol. 20:18. doi: 10.1186/s13059-019-1629-z

Long, J. M., and Holtzman, D. M. (2019). Alzheimer Disease: An update on pathobiology and treatment strategies. Cell 179, 312-339. doi: 10.1016/j.cell. 2019.09.001

Long, J., Ray, B., and Lahiri, D. (2012). MicroRNA-153 physiologically inhibits expression of amyloid- $\beta$ precursor protein in cultured human fetal brain cells and is dysregulated in a subset of Alzheimer disease patients. J. Biol. Chem. 287, 31298-31310. doi: 10.1074/jbc.M112.366336

Lugli, G., Cohen, A., Bennett, D., Shah, R., Fields, C., Hernandez, A., et al. (2015). Plasma exosomal miRNAs in persons with and without Alzheimer disease: altered expression and prospects for biomarkers. PLoS One 10:e0139233. doi: 10.1371/journal.pone.0139233

Maximov, A., Tang, J., Yang, X., Pang, Z. P., and Südhof, T. C. (2009). Complexin controls the force transfer from SNARE complexes to membranes in fusion. Science 323, 516-521. doi: 10.1126/science.1166505

McKhann, G., Knopman, D., Chertkow, H., Hyman, B., Jack, C., Kawas, C., et al. (2011). The diagnosis of dementia due to Alzheimer's disease: recommendations from the National Institute on Aging-Alzheimer's Association workgroups on diagnostic guidelines for Alzheimer's disease. Alzheimers Dement. 7, 263-269. doi: 10.1016/j.jalz.2011.03.005

Mengel-From, J., Feddersen, S., Halekoh, U., Heegaard, N. H. H., McGue, M., Christensen, K., et al. (2018). Circulating microRNAs disclose biology of normal cognitive function in healthy elderly people - a discovery twin study. Eur. J. Hum. Genet. 26, 1378-1387. doi: 10.1038/s41431-018-0157-8

Mitchell, P. S., Parkin, R. K., Kroh, E. M., Fritz, B. R., Wyman, S. K., PogosovaAgadjanyan, E. L., et al. (2008). Circulating microRNAs as stable blood-based markers for cancer detection. Proc. Natl. Acad. Sci. U.S.A. 105, 10513-10518. doi: 10.1073/pnas.0804549105

Paoletti, P., and Neyton, J. (2007). NMDA receptor subunits: function and pharmacology. Curr. Opin. Pharmacol. 7, 39-47. doi: 10.1016/j.coph.2006.08.011 
Pascual-Lucas, M., Viana da Silva, S., Di Scala, M., Garcia-Barroso, C., GonzálezAseguinolaza, G., Mulle, C., et al. (2014). Insulin-like growth factor 2 reverses memory and synaptic deficits in APP transgenic mice. EMBO Mol. Med. 6, 1246-1262. doi: $10.15252 / \mathrm{emmm} .201404228$

Portelius, E., Zetterberg, H., Skillbäck, T., Törnqvist, U., Andreasson, U., Trojanowski, J., et al. (2015). Cerebrospinal fluid neurogranin: relation to cognition and neurodegeneration in Alzheimer's disease. Brain 138, 3373-3385. doi: 10.1093/brain/awv267

Preische, O., Schultz, S. A., Apel, A., Kuhle, J., Kaeser, S. A., Barro, C., et al. (2019). Serum neurofilament dynamics predicts neurodegeneration and clinical progression in presymptomatic Alzheimer's disease. Nat. Med. 25, 277-283. doi: 10.1038/s41591-018-0304-3

Raja, M. K., Preobraschenski, J., Del Olmo-Cabrera, S., Martinez-Turrillas, R., Jahn, R., Perez-Otano, I., et al. (2019). Elevated synaptic vesicle release probability in synaptophysin/gyrin family quadruple knockouts. eLife 8:e40744. doi: 10.7554/ eLife.40744

Ritchie, M., Phipson, B., Wu, D., Hu, Y., Law, C., Shi, W., et al. (2015). limma powers differential expression analyses for RNA-sequencing and microarray studies. Nucleic Acids Res. 43, e47-e47. doi: 10.1093/nar/gkv007

Ross, S. P., Baker, K. E., Fisher, A., Hoff, L., Pak, E. S., and Murashov, A. K. (2018). miRNA-431 prevents amyloid- $\beta$-induced synapse loss in neuronal cell culture model of Alzheimer's disease by silencing kremen1. Front. Cell. Neurosci. 12:87. doi: $10.3389 /$ fncel.2018.00087

Scheff, S. W., Price, D. A., Schmitt, F. A., DeKosky, S. T., and Mufson, E. J. (2007). Synaptic alterations in CA1 in mild Alzheimer disease and mild cognitive impairment. Neurology. 68, 1501-1508. doi: 10.1212/01.wnl.0000260698.46517.8f

Selkoe, D., and Hardy, J. (2016). The amyloid hypothesis of Alzheimer's disease at 25 years. EMBO Mol. Med. 8, 595-608. doi: 10.15252/emmm.201606210

Song, Y., Hu, M., Zhang, J., Teng, Z. Q., and Chen, C. (2019). A novel mechanism of synaptic and cognitive impairments mediated via microRNA-30b in Alzheimer's disease. EBioMedicine 39, 409-421. doi: 10.1016/j.ebiom.2018. 11.059

Stanley, K., Whitfield, T., Kuchenbaecker, K., Sanders, O., Stevens, T., and Walker, Z. (2019). Rate of cognitive decline in Alzheimer's disease stratified by age. J. Alzheimers Dis. 69, 1153-1160. doi: 10.3233/JAD- 181047

Stout, K. A., Dunn, A. R., Hoffman, C., and Miller, G. W. (2019). The Synaptic Vesicle Glycoprotein 2: Structure, Function, and Disease Relevance. ACS Chem. Neurosci. 10, 3927-3938. doi: 10.1021/acschemneuro.9b 00351

Sun, X., Wu, Y., Gu, M., and Zhang, Y. (2014). miR-342-5p decreases ankyrin G levels in Alzheimer's disease transgenic mouse models. Cell Rep. 6, 264-270. doi: 10.1016/j.celrep.2013.12.028

Takousis, P., Sadlon, A., Schulz, J., Wohlers, I., Dobricic, V., Middleton, L., et al. (2019). Differential expression of microRNAs in Alzheimer's disease brain, blood, and cerebrospinal fluid. Alzheimers Dement. 15, 1468-1477. doi: 10. 1016/j.jalz.2019.06.4952

Tan, L., Yu, J., Liu, Q., Tan, M., Zhang, W., Hu, N., et al. (2014). Circulating miR-125b as a biomarker of Alzheimer's disease. J. Neurol. Sci. 336, 52-56. doi: 10.1016/j.jns.2013.10.002

Taylor, L. M., McMillan, P. J., Kraemer, B. C., and Liachko, N. F. (2019). Tau tubulin kinases in proteinopathy. FEBS J. 286, 2434-2446. doi: 10.1111/febs.14866
Turchinovich, A., Weiz, L., Langheinz, A., and Burwinkel, B. (2011). Characterization of extracellular circulating microRNA. Nucleic Acids Res. 39, 7223-7233. doi: 10.1093/nar/gkr254

Urbina, F. L., Menon, S., Goldfarb, D., Edwards, R., Ben Major, M., Brennwald, P., et al. (2021). TRIM67 regulates exocytic mode and neuronal morphogenesis via SNAP47. Cell Rep. 34:108743. doi: 10.1016/j.celrep.2021.108743

Vanlandingham, P. A., Barmchi, M. P., Royer, S., Green, R., Bao, H., Reist, N., et al. (2014). AP180 couples protein retrieval to clathrin-mediated endocytosis of synaptic vesicles. Traffic. 15, 433-450. doi: 10.1111/tra.12153

Wang, W., Kwon, E., and Tsai, L. (2012). MicroRNAs in learning, memory, and neurological diseases. Learn. Mem. 19, 359-368. doi: 10.1101/lm.026492.112

Wang, X., Liu, D., Huang, H. Z., Wang, Z. H., Hou, T. Y., Yang, X., et al. (2018). A novel microRNA-124/ptpn1 signal pathway mediates synaptic and memory deficits in Alzheimer's disease. Biol. Psychiatry. 83, 395-405. doi: 10.1016/j. biopsych.2017.07.023

Wang, Y., Okamoto, M., Schmitz, F., Hofmann, K., and Südhof, T. C. (1997). Rim is a putative Rab3 effector in regulating synaptic-vesicle fusion. Nature 388, 593-598. doi: $10.1038 / 41580$

Wiedrick, J. T., Phillips, J. I., Lusardi, T. A., McFarland, T. J., Lind, B., Sandau, U. S., et al. (2019). Validation of microRNA biomarkers for Alzheimer's disease in human cerebrospinal fluid. J. Alzheimers Dis. 67, 875-891. doi: 10.3233/JAD180539

Wu, X., Hu, S., Kang, X., and Wang, C. (2020). Synaptotagmins: Beyond Presynaptic Neurotransmitter Release. Neuroscientist 26, 9-15. doi: 10.1177/ 1073858419844497

Wylie, D., Shelton, J., Choudhary, A., and Adai, A. (2011). A novel mean-centering method for normalizing microRNA expression from high-throughput RTqPCR data. BMC Res. Notes. 4:555. doi: 10.1186/1756-0500-4-555

Yap, K., Drakew, A., Smilovic, D., Rietsche, M., Paul, M. H., Vuksic, M., et al. (2020). The actin-modulating protein synaptopodin mediates long-term survival of dendritic spines. eLife 9:e62944. doi: 10.7554/eLife.62944

Conflict of Interest: DG-C has filed a patent on miRNAs as biomarkers.

The remaining authors declare that the research was conducted in the absence of any commercial or financial relationships that could be construed as a potential conflict of interest.

Publisher's Note: All claims expressed in this article are solely those of the authors and do not necessarily represent those of their affiliated organizations, or those of the publisher, the editors and the reviewers. Any product that may be evaluated in this article, or claim that may be made by its manufacturer, is not guaranteed or endorsed by the publisher.

Copyright (C) 2021 Dakterzada, David Benitez, Targa, Lladó, Torres, Romero, de Gonzalo-Calvo, Moncusi-Moix, Tort-Merino, Huerto, Sánchez-de-la-Torre, Barbé and Piñol-Ripoll. This is an open-access article distributed under the terms of the Creative Commons Attribution License (CC BY). The use, distribution or reproduction in other forums is permitted, provided the original author(s) and the copyright owner(s) are credited and that the original publication in this journal is cited, in accordance with accepted academic practice. No use, distribution or reproduction is permitted which does not comply with these terms. 